\title{
Hardware Implementation of a Modified Delay-Coordinate Mapping-Based QRS Complex Detection Algorithm
}

\author{
Matej Cvikl,, ${ }^{1}$ Franc Jager, ${ }^{2}$ and Andrej Zemva ${ }^{3}$ \\ ${ }^{1}$ Iskra Sistemi, d.d., Stegne 21, 1000 Ljubljana, Slovenia \\ ${ }^{2}$ Laboratory of Biomedical Computer Systems and Imaging, Faculty of Computer and Information Science, \\ University of Ljubljana, Trzaska 25, 1000 Ljubljana, Slovenia \\ ${ }^{3}$ Laboratory for Integrated Circuit Design, Faculty of Electrical Engineering, University of Ljubljana, \\ Trzaska 25, 1000 Ljubljana, Slovenia
}

Received 30 April 2006; Revised 23 January 2007; Accepted 23 January 2007

Recommended by David Hamilton

\begin{abstract}
We present a modified delay-coordinate mapping-based QRS complex detection algorithm, suitable for hardware implementation. In the original algorithm, the phase-space portrait of an electrocardiogram signal is reconstructed in a two-dimensional plane using the method of delays. Geometrical properties of the obtained phase-space portrait are exploited for QRS complex detection. In our solution, a bandpass filter is used for ECG signal prefiltering and an improved method for detection threshold-level calculation is utilized. We developed the algorithm on the MIT-BIH Arrhythmia Database (sensitivity of $99.82 \%$ and positive predictivity of $99.82 \%$ ) and tested it on the long-term ST database (sensitivity of $99.72 \%$ and positive predictivity of $99.37 \%$ ). Our algorithm outperforms several well-known QRS complex detection algorithms, including the original algorithm.
\end{abstract}

Copyright (C) 2007 Matej Cvikl et al. This is an open access article distributed under the Creative Commons Attribution License, which permits unrestricted use, distribution, and reproduction in any medium, provided the original work is properly cited.

\section{INTRODUCTION}

Detecting QRS complexes is the most important task in electrocardiogram (ECG) signal analysis. Physiological variability of QRS complexes and various types of artifacts like muscle noise, power line interference, baseline wander, motion artifacts, and electrode contact noise added to the ECG signal make QRS complex detection a difficult task.

In the literature, various types of QRS complex detection algorithms can be found. Köhler et al. [1] divided QRS complex detection algorithms into algorithms based on signal derivatives and digital filters, wavelet-based QRS complex detection, neural network approaches, and additional approaches. The algorithms from the first two groups are most widely used for QRS complex detection and can be found in software (SW) or hardware (HW) implementations.

The first group encompasses algorithms based on signal derivatives and digital filters. Such approach divides the search process into two stages: the preprocessing stage and the decision stage. The preprocessing stage usually consists of a band-pass linear filter to reduce noise and enhance the QRS complex $[2,3]$, and a nonlinear filter, which by signal differentiation, squaring, and integration differentiates the
QRS complex from the artifacts. The decision stage applies QRS complex search rules where the detection function is compared to a certain detection threshold level to find signal peaks and then the decision is made whether the peak is a QRS complex or not. One of the best performing algorithms in this group is an open source ECG analysis SW (OSEA) [4]; OSEA achieves QRS complex detection sensitivity (Se) of $99.80 \%$ and positive predictivity $(+\mathrm{P})$ of $99.80 \%$ on the MIT-BIH Arrhythmia Database [5]. Further examples of algorithms with lower performance that can be classified into this group can be found in [6-10].

The variety of QRS complex shape morphologies and artifacts causes the performance of QRS complex detection algorithms that use fixed bandwidth bandpass filters and fixed width integration windows to decrease when the QRS morphology changes. To avoid this problem, a new approach to QRS complex detection based on wavelet transform (WT) has been introduced. The WT decomposes the ECG signal into several scales, where each scale has different bandwidth and time support. WT at any scale is done by filtering the signal with an appropriate filter. The most common approach to QRS complex detection is finding local maxima at four consecutive scales. Detection starts at the largest scale $\left(W_{2}{ }^{4}\right)$ 


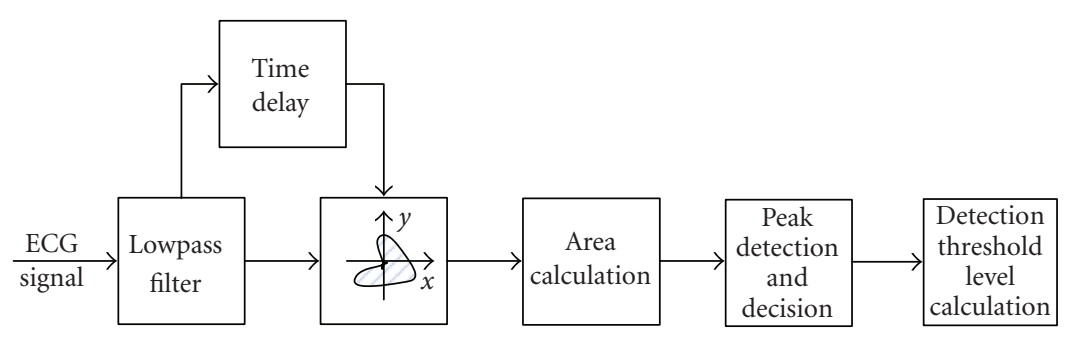

FIGURE 1: QRS complex detection using delay-coordinate mapping [11].

containing low-frequency signal information and continues to the lowest scale $\left(W_{2}{ }^{1}\right)$ containing high-frequency signal information. A positive-negative pair of local maxima that occurs at all the scales at the same time and meets other detection rules is declared as the QRS complex. One of the first WT-based QRS complex detection algorithms with very high Se of $99.89 \%$ and $+\mathrm{P}$ of $99.94 \%$ tested on the MIT-BIH Arrhythmia Database was published by Li et al. [12]. This algorithm serves as a starting point for other algorithms which usually only differ in the usage of different wavelet functions [13], number of processed scales [14], or modifications to the original algorithm $[15,16]$. These algorithms also have lower performance than [12].

While algorithms from the first group are usually less computationally intensive and suitable for microcontroller implementations in embedded systems, other types of algorithms usually utilize a greater number of filters and decision rules, making them unsuitable for systems with low-signal processing power. Most of such algorithms run on personal computers (PCs) where data is processed further. Regardless of the type of the algorithm, when a lot of ECG signals need to be processed on a single PC in a relatively short time, it is essential to have enough processing power. All the signals need to be processed without degrading the performance of other applications running on the PC. One solution to the problem is upgrading the PC, while the second solution is in an application-specific HW. Such HW can be based on a digital signal processor (DSP) or field programmable gate array (FPGA) added to the PC, so the processor in the PC can be relieved from most of the computation.

The goal of our research work was development and HW implementation of a QRS complex detection algorithm in order to set the groundwork for further ECG signal processing stages in HW. Because of expected high-speed data processing, the HW is aimed to be used as a QRS complex detection engine in systems where a larger number of ECG signals have to be processed. In this paper, a HW implementation of the QRS complex detection algorithm based on phase-space portrait of the ECG signal is described. In contrast to the original algorithm [11], our algorithm processes blocks of ECG data, has a different input filter, and uses a different method for detection threshold level calculation. The algorithm was developed on the MIT-BIH Arrhythmia Database and a comparison with some of the well-known QRS complex detection algorithms in terms of QRS detection performance on this database was made. Finally, the algorithm was tested on the long-term ST database (LTST DB) [17].

\section{MATERIALS AND METHODS}

A real-time QRS complex detection algorithm for microcontroller implementation was adapted for block data processing in HW. The developed algorithm was first modeled and tested in SW (Matlab). The SW model served as an optimization and testing tool for the HW implementation of the algorithm, which was entirely written in very high-speed integrated circuit hardware description language (VHDL) [18].

The QRS complex detection algorithm proposed by Lee et al. [11] uses time delay to map the input ECG signal. A single ECG signal and its time delayed duplicate create a phasespace portrait, whose geometrical properties are exploited for QRS complex detection. Based on the size of the polygon bounded by a number of consecutive data points, a QRS event can be found. Each time a new polygon size (area) exceeding the current detection threshold level is found, a decision is made whether the peak is indeed a QRS complex or not, and the detection threshold level is updated. A block diagram of the algorithm is shown in Figure 1.

Although this scheme requires several multiplications for area calculation, the algorithm is relatively simple and has satisfactory performance: Se of $99.69 \%$ and $+\mathrm{P}$ of $99.88 \%$, tested on the MIT-BIH Arrhythmia Database. In order to simplify the preprocessing stage, authors deliberately used only a lowpass filter, which resulted in more dynamic changes of the polygon sizes since $\mathrm{P}$ and $\mathrm{T}$ waves and motion artifacts were not filtered out.

We modified the algorithm in order to make it more suitable for postprocessing, where the ECG signal is processed block-by-block. It is designed and optimized for QRS complex detection in the ECG signal sampled at $250 \mathrm{~Hz}$. However, other sampling rates can be used with appropriate adjustments to the input filter, correct block length, and detection threshold level calculation. The algorithm we propose is shown in Figure 2.

As can be seen in Figure 2, the modified algorithm comprises a bandpass filter instead of the lowpass filter, and the detection threshold level calculation is performed before peak detection. Moreover, a different method for the detection threshold level calculation is utilized. The algorithm is described in more detail in the following subsections. 


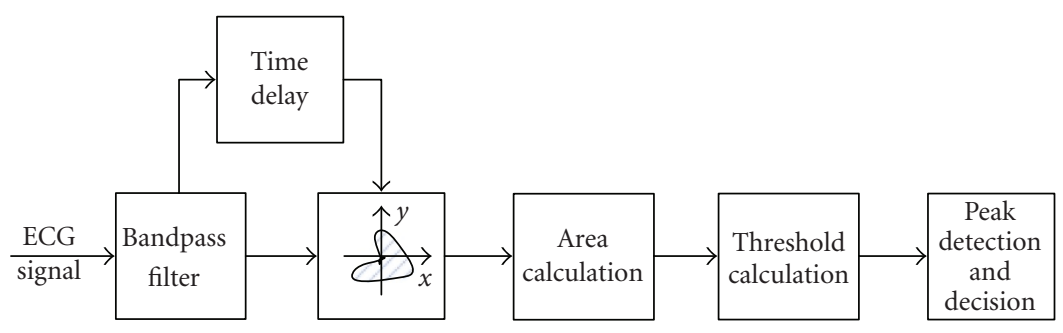

FIgURE 2: Modified delay-coordinate mapping algorithm.

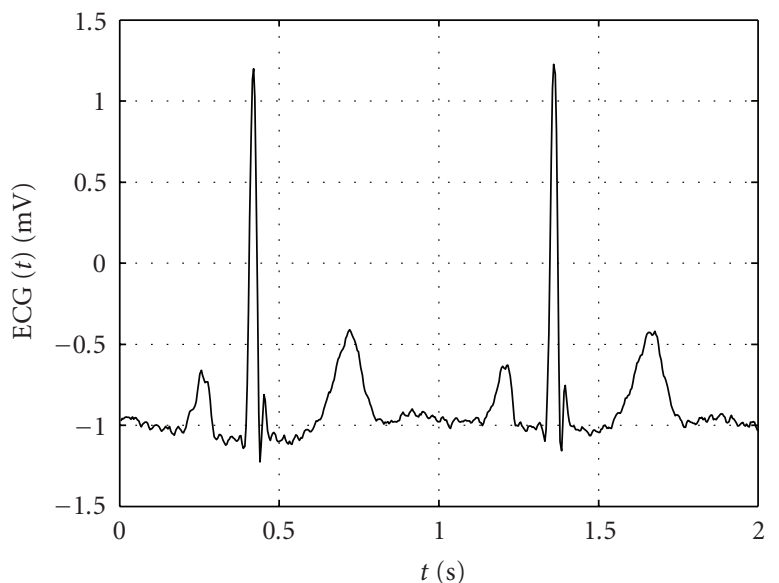

(a)

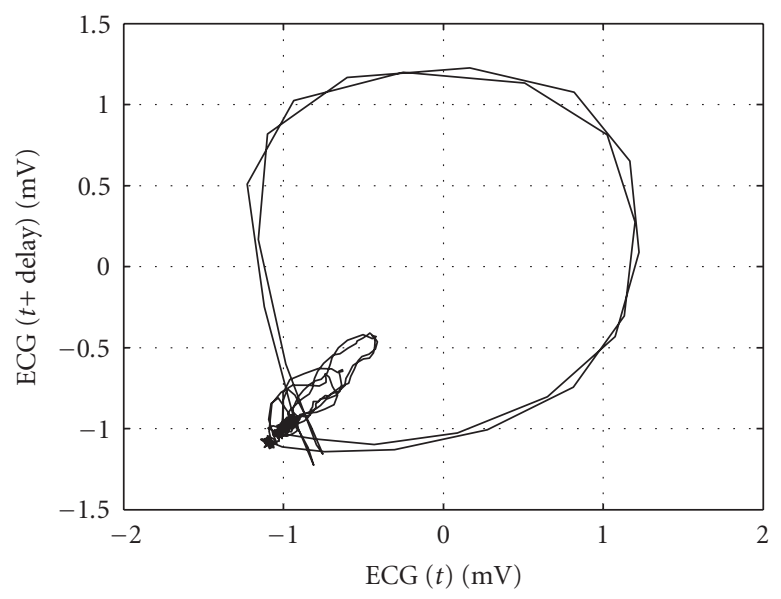

(c)

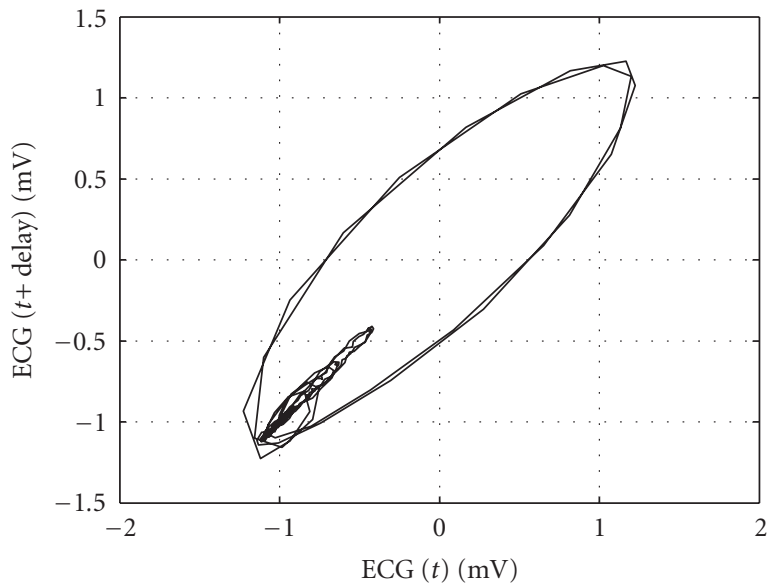

(b)

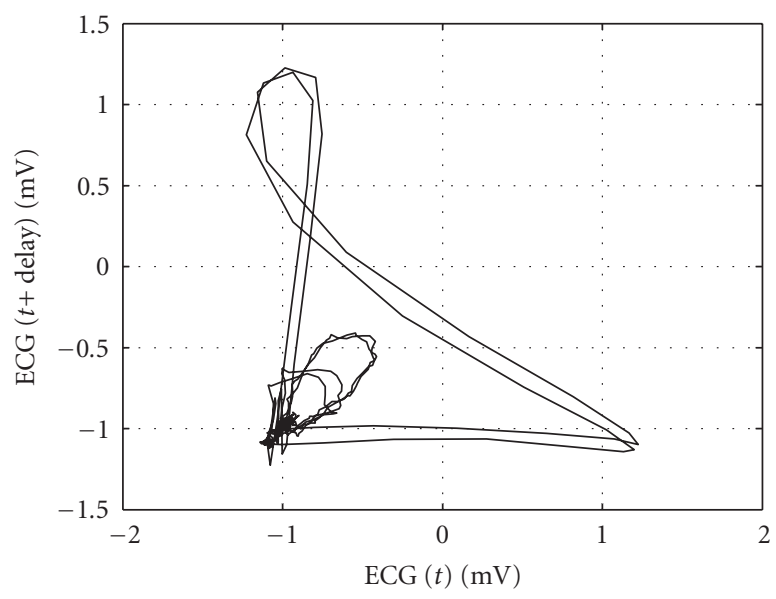

(d)

FIgUre 3: Phase-space portraits of the ECG signal: (a) input ECG signal, (b) phase-space portrait of the ECG signal with the time delay of 4 milliseconds, (c) phase-space portrait of the ECG signal with the time delay of 12 milliseconds, (d) phase-space portrait of the ECG signal with the time delay 28 milliseconds.

\subsection{Phase-space portrait}

The phase-space portrait (also phase portrait) of a signal is constructed in a $2 \mathrm{D}$ plane $(x-y)$ from the original signal and its time delayed duplicate, so that coordinates of each data point are written as $x[n \mathrm{~T}]=\operatorname{ECG}[n \mathrm{~T}]$ and $y[n \mathrm{~T}]=\operatorname{ECG}[(n-\tau) \mathrm{T}]$, where $\tau$ is the time delay. Figure 3 shows exemplary phase-space portraits of two consecutive heartbeats, where trajectories are generated by delaying the signal by 4 milliseconds, 12 milliseconds, and 28 milliseconds. In the phase-space portraits, we can distinct three different areas which the trajectory forms in the $2 \mathrm{D}$ plane: the smaller areas are created by the $\mathrm{P}$ and $\mathrm{T}$ waves with a lower amplitude, while the largest area corresponds to a higher amplitude wave or the QRS complex, respectively. 


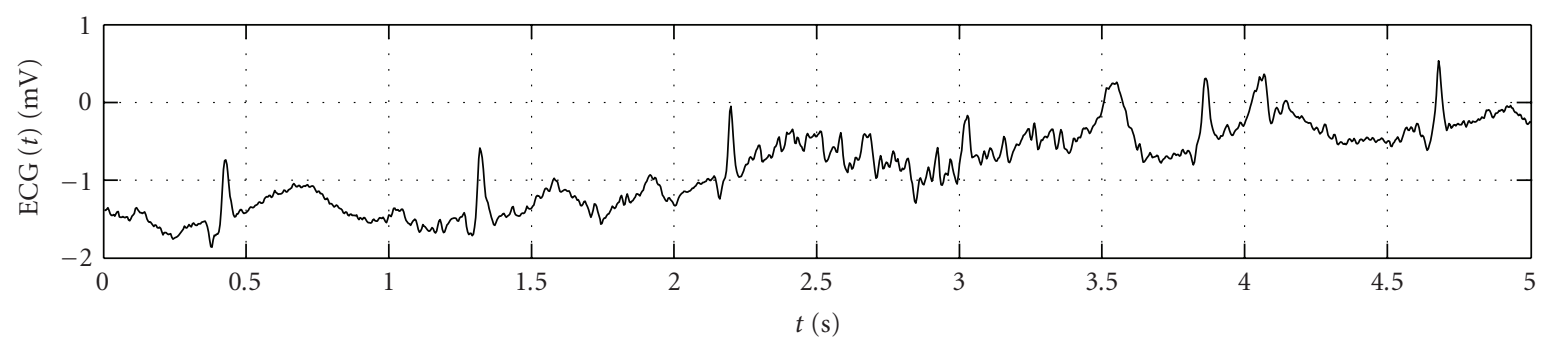

(a)

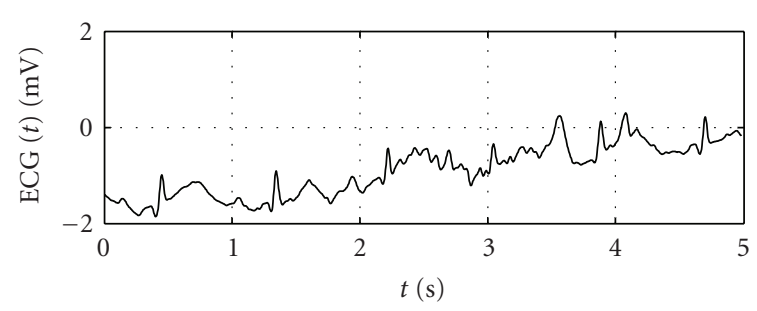

(b)

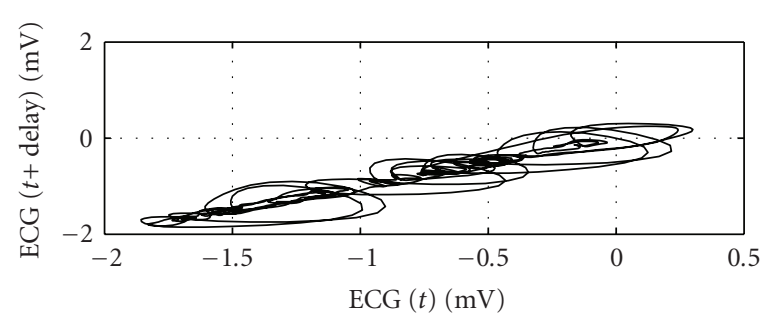

(d)

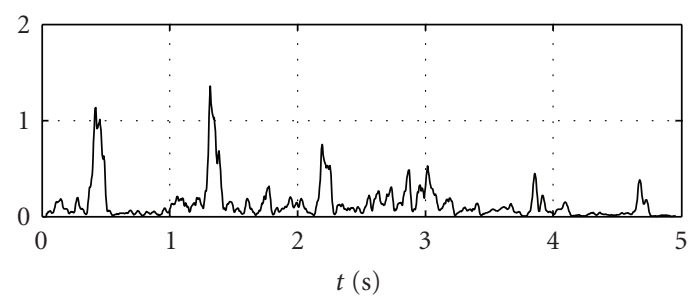

(f)

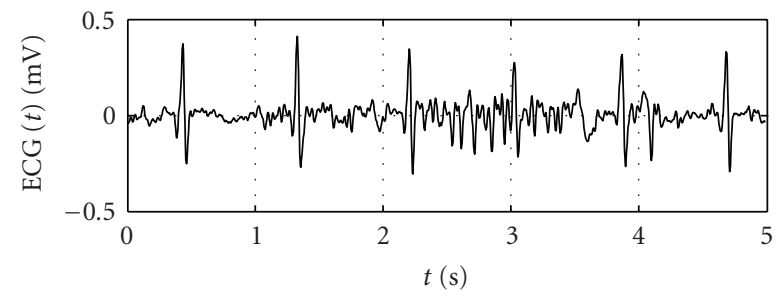

(c)

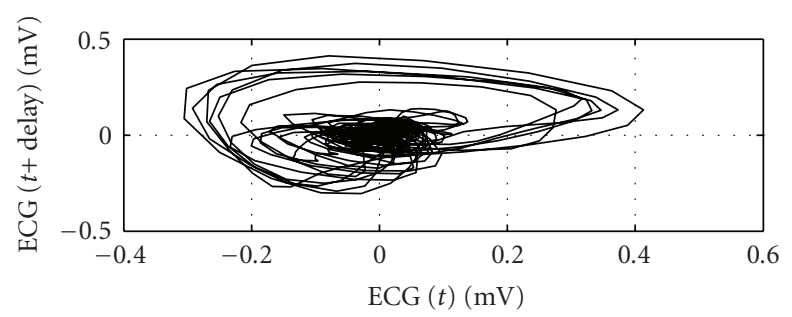

(e)

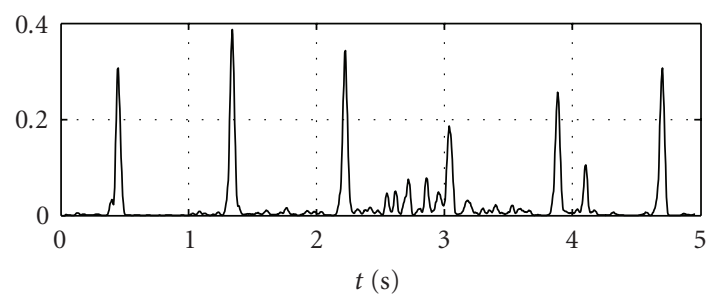

(g)

FIGURE 4: Phase-space portraits and the calculated area of the ECG signal: (a) input ECG signal, (b) lowpass filtered ECG signal, (c) bandpass filtered ECG signal, (d) phase-space portrait of the lowpass filtered ECG signal, (e) phase-space portrait of the bandpass filtered ECG signal, (f) calculated area of the lowpass filtered ECG signal, (g) calculated area of the bandpass filtered ECG signal.

It is very important that an appropriate signal time delay is chosen in order to get useful phase-space portraits and to sustain proportions between the sizes of areas created by different waves. If the time delay is too short (Figure 3(b)), the resulting trajectory creates rather "flat" areas around the $y=x$ line, that can potentially be disordered, but on the other hand, if the time delay is too great (Figure 3(d)), the contour can create several smaller areas instead of one larger area. Based on their QRS detection algorithm performance, Lee et al. [11] determined that the optimal time delay is 20 milliseconds.

Some phase-space portrait examples of ECG signals contaminated with high-frequency noise and baseline drifts are shown in [11]. While baseline drifts cause the phase-space portrait to move along the diagonal axis of the plane, along the $y=x$ line, and do not influence the size of the area itself, the high-frequency noise can distort the phase-space portrait to a point where the distinctive area created by the QRS complex cannot be found anymore. To avoid this, the ECG signal is to be prefiltered, so the phase-space portrait is constructed from the filtered ECG signal and its time delayed duplicate. Figure 4 shows the input ECG signal, lowpass, and bandpass filtered signals, their phase-space portraits, and sizes of the areas the trajectories created. The input signal is deliberately selected to emphasize the difference between the phase-space portraits created by the two filters.

When the signal is filtered with the lowpass filter, the influence of baseline drift can be seen; the trajectory created 
by time delaying the signal moves along the $y=x$ line (Figure 4(d)), while no influence can be seen on the calculated area (Figure 4(f)). It can also be noted that the lowpass filter allows waves like $\mathrm{P}$ and $\mathrm{T}$ to create small areas (Figure 4(f)), which add to more dynamical area behavior. If the ECG signal is filtered with a bandpass filter, the trajectory does not move along the $y=x$ line (Figure 4(e)) and there are hardly any areas present that would be created by the $\mathrm{P}$ and $\mathrm{T}$ waves, as these waves are attenuated (Figure $4(\mathrm{~g})$ ). It can also be noticed that the different frequency characteristics of the bandpass filter result in a different shape and position of the shape the trajectory tends to form in the $2 \mathrm{D}$ plane (Figures 4(d), 4(e)).

\subsection{Area calculation}

As mentioned in the previous subsection, each data point in the $x-y$ plane has coordinates $(x[n \mathrm{~T}], y[n \mathrm{~T}])$. The size of an area formed by $n$ data points ( $n$-point data vector) is used as the detection function for locating QRS complexes. The area is obtained using plane geometry equation for a planar non-self-intersecting polygon area calculation:

$$
\text { Area }=\frac{1}{2}\left(\left|\begin{array}{ll}
x_{1} & x_{2} \\
y_{1} & y_{2}
\end{array}\right|+\left|\begin{array}{ll}
x_{2} & x_{3} \\
y_{2} & y_{3}
\end{array}\right|+\cdots+\left|\begin{array}{ll}
x_{n-1} & x_{n} \\
y_{n-1} & y_{n}
\end{array}\right|\right) .
$$

Polygon orientation is not important, so the absolute value of the calculated area (determinant) has to be considered. Lee et al. [11] calculated the sizes of areas comprised of ten data points. Their decision was based on the fact that if the average QRS complex duration is less than 100 milliseconds (25 data points at 250 samples per second), all polygons comprised of ten data points are nonintersecting. The experiment for obtaining the optimum number of data points for our algorithm is described in Section 3.

\subsection{Filtering}

Targeted for HW implementation in FPGA, the chosen filter belongs to a family of simple nonrecursive filters with integer multipliers $[19,20]$. Impulse response of the filter used in this work is shown in Figure 5(a). The filter comprises two sections, where each section of length $L$ calculates the average value of $L$ neighboring data samples; thus each section represents a moving average filter. By averaging consecutive data samples, the high-frequency components of the input signal are attenuated. To attenuate power-line interference, the length $L$ of the two sections is calculated so that the filter has zero gain at $50 \mathrm{~Hz}(f 50)$ and multiples of $50 \mathrm{~Hz}$. For $250 \mathrm{~Hz}$ sampling rate $(f s)$, the section length $L$ is five:

$$
L=\frac{f s}{f_{50}} .
$$

The filter can be redesigned for other sampling rates accordingly, again attenuating $50 \mathrm{~Hz}$. Furthermore, the impulse response of the filter was chosen in such a way that there is a step between coefficient values. This results in emphasis on high-speed transitions in the input ECG signal, that

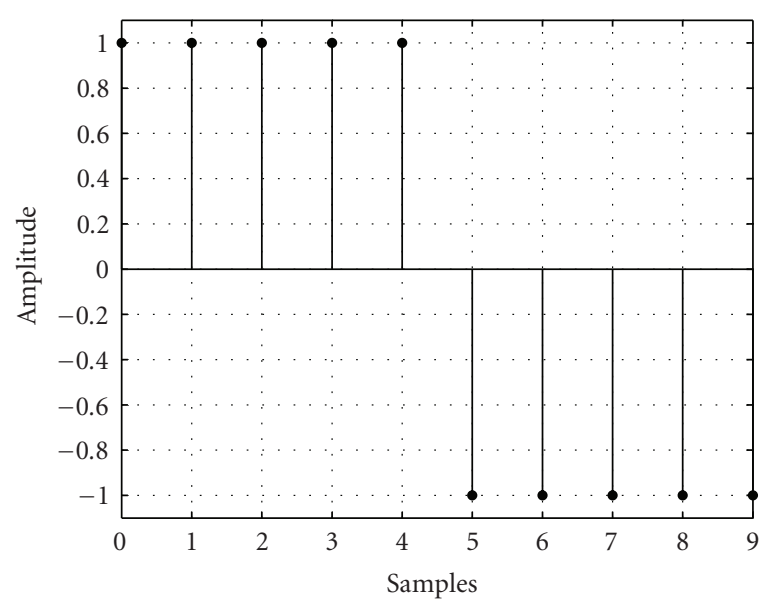

(a)

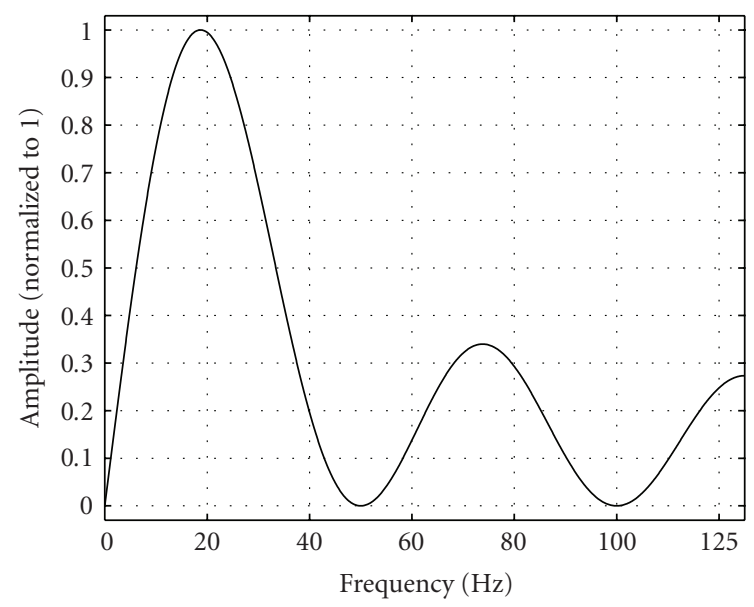

(b)

Figure 5: Filter characteristics: (a) impulse response, (b) frequency response.

is, slopes of the QRS complex, and attenuation of the lowfrequency components. Frequency response of the filter is shown in Figure 5(b). The difference equation of the filter is the following:

$$
\begin{aligned}
y[n]= & x[n]+x[n-1]+x[n-2]+x[n-3] \\
& +x[n-4]-x[n-5]-x[n-6] \\
& -x[n-7]-x[n-8]-x[n-9] .
\end{aligned}
$$

Frequency response of the filter shows attenuation of lowand high-frequency components.

We can also see approximately linear characteristic below the center frequency yielding sensitivity of the bandpass filter to slopes in original signal and cancelation of the $50 \mathrm{~Hz}$ component and its multiples. The center frequency of the filter designed is at $18.7 \mathrm{~Hz}$ and its cutoff frequencies are at $9.2 \mathrm{~Hz}$ and $29.3 \mathrm{~Hz}$.

An example of an input ECG signal and the filtered signal is shown in Figure 6. It is clearly visible that faster slopes in the original signal were emphasized or extracted, while 


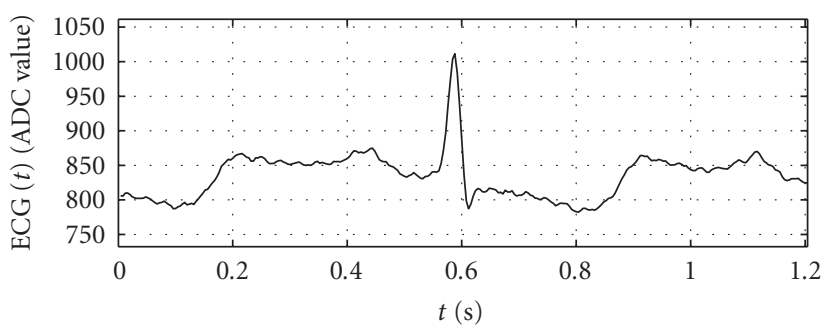

(a)

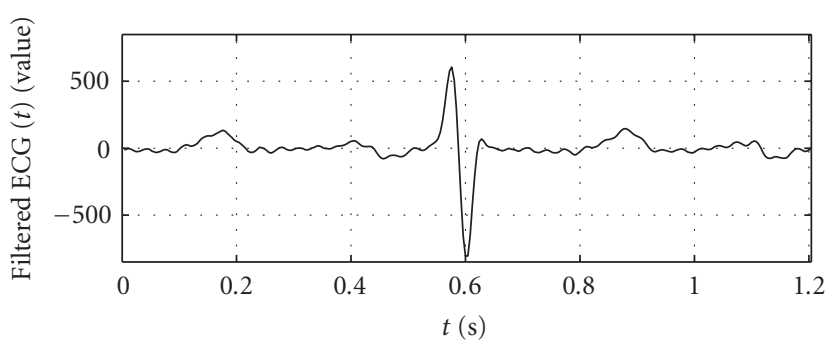

(b)

FIGURE 6: Original ECG signal and filtered ECG signal.

slowly varying waves like $\mathrm{P}$ wave, ST segment, and T wave composed from low frequencies were attenuated.

\subsection{Detection threshold level calculation}

The bandpass filter significantly reduces the influence of lower- and higher-frequency components on the calculated area, which can be seen when Figures 4(f) and 4(g) are compared. In Figure 4(g), representing the areas created by the bandpass filtered signal, the peaks produced by the QRS complexes can clearly be distinguished from other peaks. This is true even when the signal is noisy, like it is between the third and the fourth heartbeats in Figure 4. The reduction of areas created by non-QRS artifacts enables a different approach to the QRS complex detection threshold level calculation than what was originally used in [11]. Our detection threshold level evaluation is based on the average value of the detection function in the current data block. As the average value of the calculated areas in the block was not high enough to filter out the peaks that were not due to QRS complexes, multiplied average values were tested. After extensive testing, the optimal value to be used as a detection threshold level was determined to be four times the average area value in the data block.

Two additional safety mechanisms for error prevention were incorporated in our algorithm and are depicted in Figure 7.

The first mechanism assures that blocks, where the calculated detection threshold level is too low, are treated as blocks with no QRS. Without this fail-safe, in blocks with no QRS complexes, a peak of any height would be recognized as a QRS complex and the number of false positive (FP) detections would increase. A search was conducted to find the optimum lower limiting value that can be represented

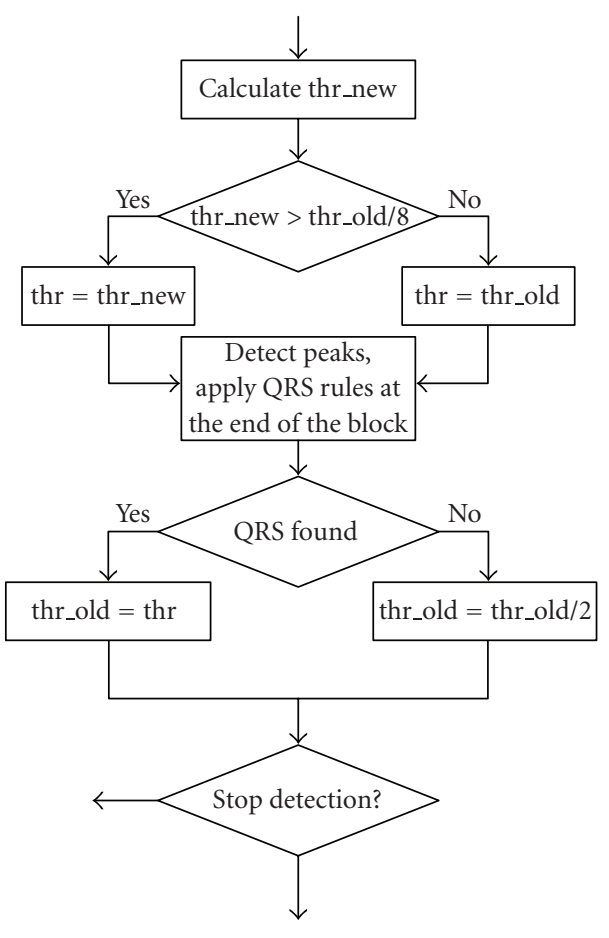

FIGURE 7: Scheme of QRS complex detection threshold level adaptation.

with a combination of numbers of power of two. This limitation was set to avoid divisions in HW. Testing results indicated that the detection threshold level in the current block (thr_new) has to be greater than $1 / 8$ th of the threshold level in the previous block (thr_old). If this is not the case, the old value is kept as a valid detection threshold level for the current block, otherwise the new value is used and the old one is updated. The second mechanism serves for recovery from false negative (FN) detections, as each time no QRS complex is found, the old detection threshold level (thr_old) is decreased by $50 \%$. This proves to be useful in cases when nondetected weak QRS complexes are present; each time no QRS is detected, the detection threshold level (thr_old) is lowered, and eventually becomes sufficiently low to start detecting the QRS complexes. However, there is a limit to the number of the detection threshold level decreases. The algorithm only allows three consecutive detection threshold level decreases, meaning that the detection threshold level can drop to $1 / 8$ th of its initial value. The detection threshold level remains unchanged for all subsequent consecutive blocks with no QRS complexes.

\subsection{Peak search and QRS complex detection rules}

QRS complex detection is based on a set of amplitude and timing criteria widely used in detection algorithms and is shown in Figure 9(a). After the detection threshold level (thr) is set for the calculated areas, the block of the calculated areas is searched for all peaks above thr and all peaks between thr/2 and thr. All peaks that exceed thr are automatically treated as 
Error at 600 samples, 8 points

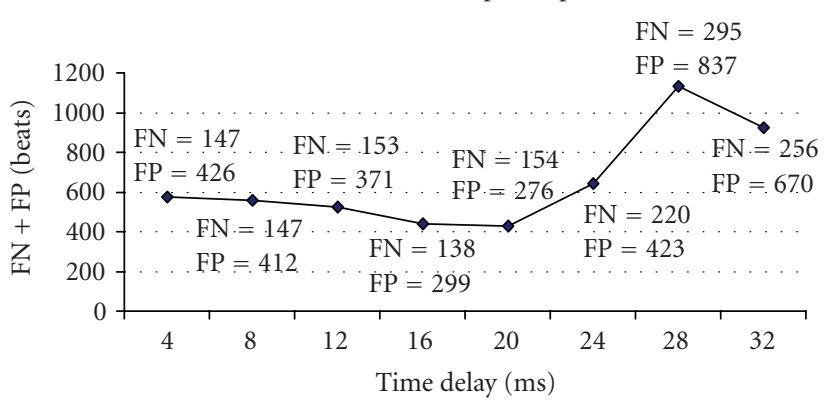

(a)

Error at 20 ms delay, 8 points

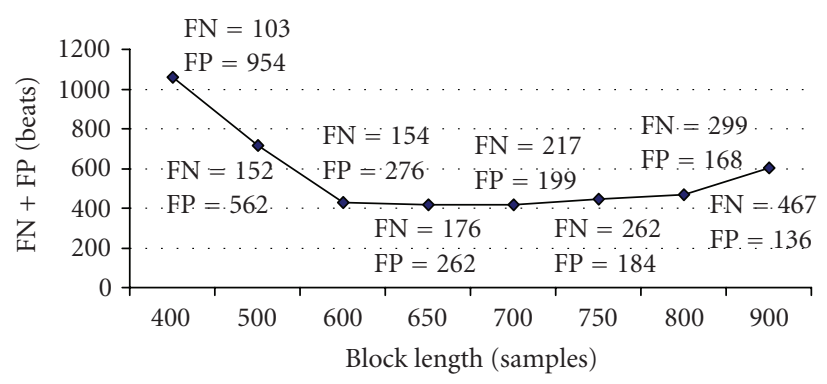

(b)

Error at 600 samples, $20 \mathrm{~ms}$ delay

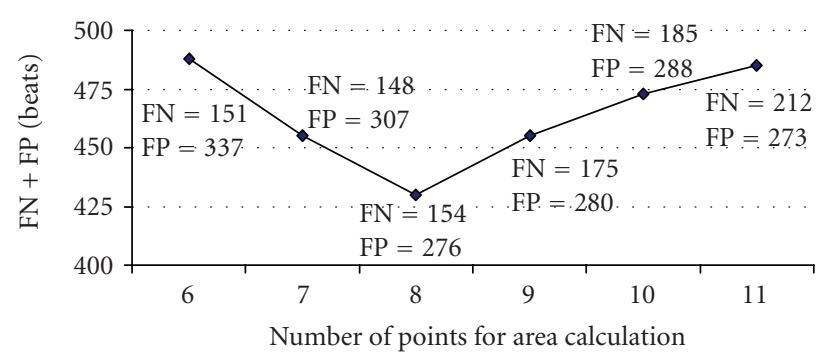

(c)

Figure 8: Influence of detection parameters ((a) time delay, (b) block length, (c) number of polygon points) on the number of missed and falsely detected beats.

QRS candidates, while the peaks between thr/2 and thr are stored to a separate list (half_peaks). Every time a new QRS candidate is found, the half_peaks list is cleared. If no QRS candidates are found within $150 \%$ of the last RR interval, the peaks from the half_peaks list are added to QRS candidates, and the half_peaks list is cleared. This procedure corresponds to the "search-back" procedure. After the complete block of the area values is swept and the QRS candidate list is obtained, QRS decision rules are applied to the list.

QRS complex decision is based on the peak mutual distance and amplitude criteria. A sweep through the peaks in the detection function is performed. The position and height of every peak in the QRS candidate list are compared to the position and height of the last known peak in the list that was recognized to originate from the QRS complex. The first peak in the candidate list is automatically recognized as the QRS complex. If the distance between the first and the second peaks is less than the refractory period (200 milliseconds), the higher of the two peaks is recognized as a QRS complex. In case the second peak is higher, the first peak is discharged and the second one is recognized as the first QRS complex. If the distance is greater than the refractory period, the second peak is automatically recognized as a new QRS complex. The third peak would then be compared to the first or the second peak, depending on which peak was recognized as the QRS complex.

After all QRS complexes in the block have been located, the difference between the last two QRS complexes is considered to be the RR interval. If none or only one QRS complex is detected in a block, the RR interval remains unchanged. It is also possible to calculate the RR interval as the mean value of all RR intervals within one block. The performance testing showed that the latter method performed worse, for which its error persistence is to be blamed. If one FP beat is detected, it takes several true positive (TP) detections to nullify its influence. Since such errors can persist through several blocks, the error can increase even further. Every FP detection causes the search-back to be performed sooner than it should, which can lead to additional FP detections. The opposite situation is also possible in cases when a few QRS complexes are missed (FN); the search-back interval can be increased and the search-back seldom performed. This way, even more FN detections can occur when the QRS complexes have their amplitude below the detection threshold level.

An additional feature of the QRS complex detection part of the algorithm is the mechanism for block overlapping. While in [13] consecutive blocks of data are overlapped by $75 \%$, our algorithm uses the last detected QRS complex in a block as a starting point for its subsequent block. The method in [13] thus produces a constant $25 \%$ processing overhead as $25 \%$ of the data is always processed twice. The overhead in our method is variable and spans between $6.7 \%$ (record 234) and 46.8\% (record 231), but is with its average value of $19.1 \%$ lower than in [13]. The same mechanism also sets the starting point when no QRS is found in the processed block. In such cases, the new starting point of the following block is set at 450 samples of the currently processed block. This means that the new block comprises 250 samples of the old data (one second of the ECG signal) and 450 samples of the new data. In cases where low-amplitude QRS complexes are present but not detected, such block composition reduces the number of FN detections, as some portions of blocks are always processed twice and each time with a lower detection threshold level.

To summarize, the main differences between the original algorithm [11] and the proposed algorithm are the following. The influence of the bandpass filter on noise and lowfrequency signals causes the calculated phase-space portrait area to be smoother. Additionally, the ECG signal is processed block-by-block, where block overlapping guarantees detection of QRS complexes that would normally be split into two separate blocks. These two features make it possible 


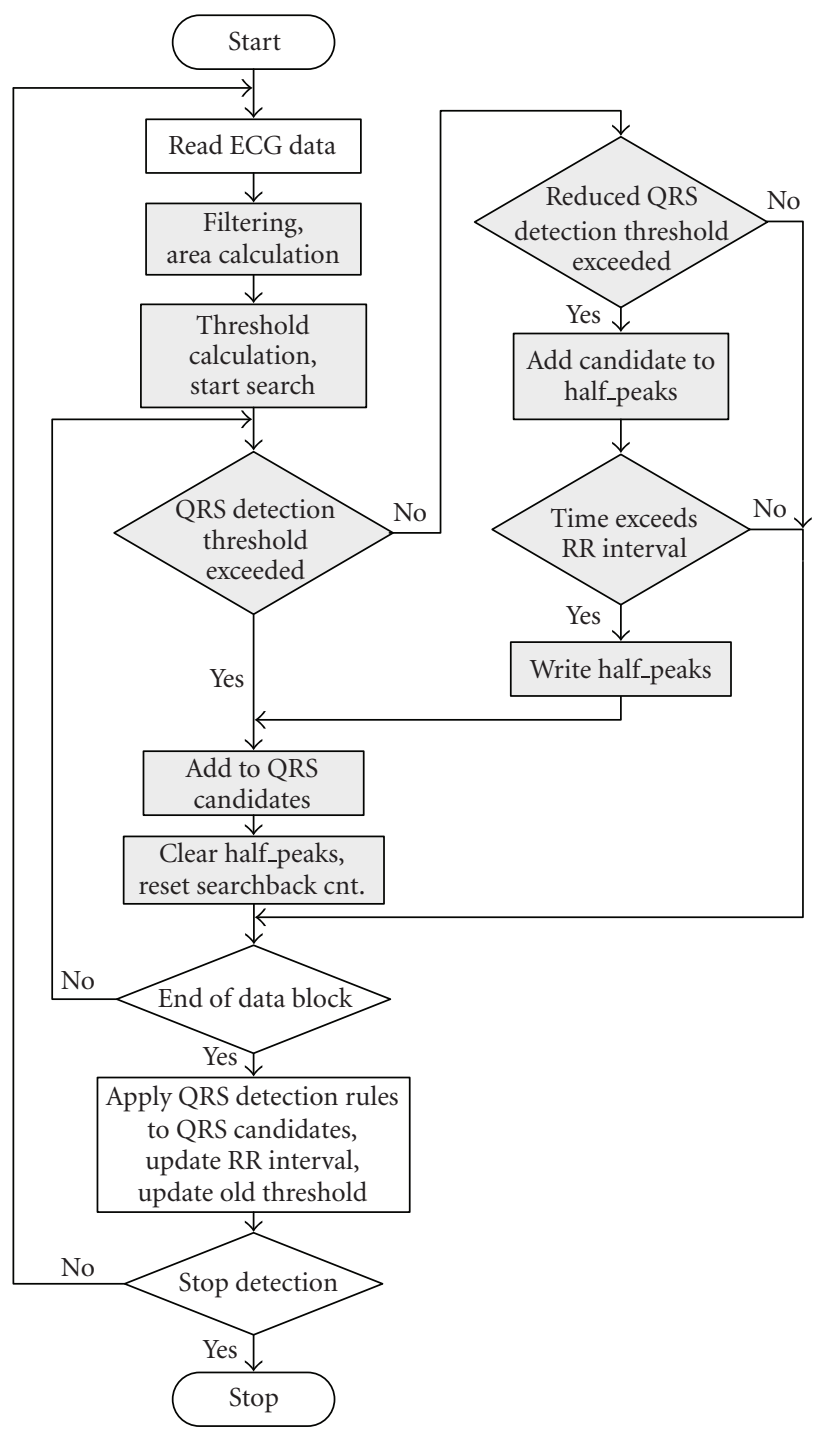

(a)

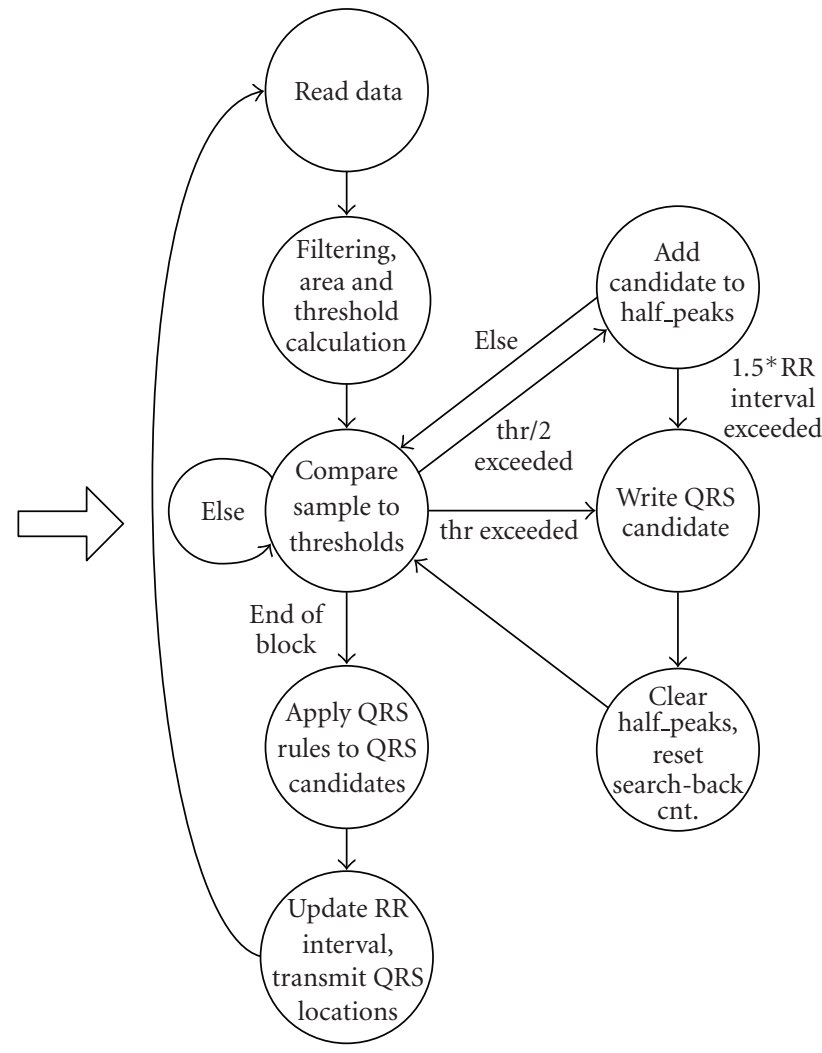

(b)

FIGURE 9: QRS complex detection algorithm: (a) flow chart of the SW implementation, (b) simplified state diagram of the HW implementation.

to calculate the QRS detection threshold level on the basis of the average value of the detection function in the current data block.

\section{IMPLEMENTATION}

The algorithm performance is highly dependant on the time delay parameter $\tau$, data block length settings, and the chosen number of polygon points. For testing purposes, the algorithm was first implemented in Matlab, where individual detection parameters were set to give optimal QRS complex detection performance in terms of the minimum number of missed and falsely detected (FN and FP) QRS complexes.

For each of the three parameters, the algorithm performance was tested on the complete database. Each test had two parameters fixed and the third parameter was chang- ing (Figure 8). First, the time delay parameter selection was made based on QRS complex detection performance with blocks of 600 data samples and 8 data points for area calculation (Figure 8(a)). In the next step, the block length was modified using the selected time delay and the same number of polygon data points (Figure 8(b)). Finally, the optimum number of data points used for area calculation was determined using 20 milliseconds delay and block length of 600 data samples (Figure 8(c)). Parameter settings with the least detection error rate were chosen for the implementation and algorithm performance was evaluated on the MIT-BIH Arrhythmia Database. The time delay was set to 20 milliseconds, the block length was set to 700 samples, and the number of points was set to eight.

Based on the SW implementation in Figure 9(a), a statemachine adapted copy of the algorithm shown in Figure 9(b) 
was developed in VHDL. The main difference between the HW implementation and the SW implementation is true parallel execution that the HW implementation offers.

All grayed parts of the code in Figure 9(a) are in the HW implementation executed independent of each other. The SW implementation needs to wait for the outcome of the detection threshold level comparison, while in the HW implementation both detection threshold level comparisons (thr and thr/2) are performed at the same time. Such scheduling significantly reduces the number of clock cycles needed for data processing and improves data processing speed. Furthermore, the average value can be calculated in parallel with area calculation, which also speeds up block processing.

Another difference in the HW implementation is that in the entire design only integer arithmetic is used. The decision to represent the variables with 32 bits or less was made to reach a compromise between the entered calculation error and resource utilization. Any variable that requires a greater number of bits is appropriately modified. Such modification is only needed at average value calculation, when 700 30-bit area size variables are to be summed. The HW divider used for average value calculation can only accept dividends of up to 32 bits; therefore, before addition, each area value is divided by 256 to achieve a 32-bit sum (dividend). Because of the high area values, such division has no influence on the detection accuracy, and does not also influence the processing speed, since divisions with the power of two are in HW only bit-shifts and zero padding.

The HW implementation of the algorithm is designed to accept the input data of a single ECG signal as a nonrepeating stream, meaning that the input logic only accepts as much data as needed to fill up the input buffer; if 200 samples of a block need to get overlapped with the next data block, only 500 additional input samples are added to construct the next data block. The resulting QRS complex detection algorithm in Figure 9(b) was implemented and tested with the Xilinx XC2VP7 [24] development board. To prove the concept, an addition of a soft-core microcontroller and a serial port interface were chosen for data transfer handling between the development board and a PC.

\section{RESULTS AND DISCUSSION}

The algorithm was developed on resampled data from the MIT-BIH Arrhythmia Database. The database contains 48 half-hour ambulatory records. These records include complex ventricular, junctional, and supraventricular arrhythmias and conduction abnormalities. Several of these records have interesting rhythm features, QRS morphology variations, and variety of changes in signal quality, thus representing "real-world" clinical conditions that may present difficulty to Arrhythmia detectors.

We tested standard performance measures such as the sensitivity $(\mathrm{Se})$ and the positive predictivity $(+\mathrm{P})$. The Se reports the percentage of true beats that were correctly detected by the algorithm, while the $+\mathrm{P}$ reports the percentage of beat detections which were in reality true beats. The test results presented in Tables 1 and 2 reflect the QRS complex detection
TABle 1: Performance of the algorithm on the MIT-BIH Arrhythmia Database.

\begin{tabular}{|c|c|c|c|c|c|c|c|}
\hline REC & AHB & $\mathrm{TP}$ & FN & $\mathrm{FP}$ & $\mathrm{FN}+\mathrm{FP}$ & Se $[\%]$ & $+\mathrm{P}[\%]$ \\
\hline 100 & 2273 & 2273 & 0 & 0 & 0 & 100.00 & 100.00 \\
\hline 101 & 1865 & 1864 & 1 & 4 & 5 & 99.95 & 99.79 \\
\hline 102 & 2187 & 2187 & 0 & 0 & 0 & 100.00 & 100.00 \\
\hline 103 & 2084 & 2084 & 0 & 0 & 0 & 100.00 & 100.00 \\
\hline 104 & 2229 & 2221 & 8 & 14 & 22 & 99.64 & 99.37 \\
\hline 105 & 2572 & 2567 & 5 & 34 & 39 & 99.81 & 98.69 \\
\hline 106 & 2027 & 2026 & 1 & 0 & 1 & 99.95 & 100.00 \\
\hline 107 & 2137 & 2135 & 2 & 0 & 2 & 99.91 & 100.00 \\
\hline 108 & 1763 & 1761 & 2 & 85 & 87 & 99.89 & 95.40 \\
\hline 109 & 2532 & 2529 & 3 & 0 & 3 & 99.88 & 100.00 \\
\hline 111 & 2124 & 2123 & 1 & 0 & 1 & 99.95 & 100.00 \\
\hline 112 & 2539 & 2539 & 0 & 0 & 0 & 100.00 & 100.00 \\
\hline 113 & 1795 & 1795 & 0 & 0 & 0 & 100.00 & 100.00 \\
\hline 114 & 1879 & 1871 & 8 & 5 & 13 & 99.57 & 99.73 \\
\hline 115 & 1953 & 1953 & 0 & 0 & 0 & 100.00 & 100.00 \\
\hline 116 & 2412 & 2393 & 19 & 3 & 22 & 99.21 & 99.87 \\
\hline 117 & 1535 & 1535 & 0 & 0 & 0 & 100.00 & 100.00 \\
\hline 118 & 2278 & 2278 & 0 & 0 & 0 & 100.00 & 100.00 \\
\hline 119 & 1987 & 1987 & 0 & 0 & 0 & 100.00 & 100.00 \\
\hline 121 & 1863 & 1862 & 1 & 1 & 2 & 99.95 & 99.95 \\
\hline 122 & 2476 & 2476 & 0 & 0 & 0 & 100.00 & 100.00 \\
\hline 123 & 1518 & 1517 & 1 & 0 & 1 & 99.93 & 100.00 \\
\hline 124 & 1619 & 1619 & 0 & 0 & 0 & 100.00 & 100.00 \\
\hline 200 & 2601 & 2599 & 2 & 3 & 5 & 99.92 & 99.88 \\
\hline 201 & 1963 & 1952 & 11 & 2 & 13 & 99.44 & 99.90 \\
\hline 202 & 2136 & 2134 & 2 & 0 & 2 & 99.91 & 100.00 \\
\hline 203 & 2980 & 2925 & 55 & 18 & 73 & 98.15 & 99.39 \\
\hline 205 & 2656 & 2653 & 3 & 0 & 3 & 99.89 & 100.00 \\
\hline 207 & 1860 & 1857 & 3 & 5 & 8 & 99.84 & 99.73 \\
\hline 208 & 2955 & 2942 & 13 & 3 & 16 & 99.56 & 99.90 \\
\hline 209 & 3005 & 3005 & 0 & 0 & 0 & 100.00 & 100.00 \\
\hline 210 & 2650 & 2633 & 17 & 4 & 21 & 99.36 & 99.85 \\
\hline 212 & 2748 & 2748 & 0 & 0 & 0 & 100.00 & 100.00 \\
\hline 213 & 3251 & 3250 & 1 & 0 & 1 & 99.97 & 100.00 \\
\hline 214 & 2262 & 2259 & 3 & 0 & 3 & 99.87 & 100.00 \\
\hline 215 & 3363 & 3363 & 0 & 0 & 0 & 100.00 & 100.00 \\
\hline 217 & 2208 & 2206 & 2 & 1 & 3 & 99.91 & 99.95 \\
\hline 219 & 2154 & 2154 & 0 & 0 & 0 & 100.00 & 100.00 \\
\hline 220 & 2048 & 2047 & 1 & 0 & 1 & 99.95 & 100.00 \\
\hline 221 & 2427 & 2416 & 11 & 0 & 11 & 99.55 & 100.00 \\
\hline 222 & 2483 & 2477 & 6 & 1 & 7 & 99.76 & 99.96 \\
\hline 223 & 2605 & 2603 & 2 & 0 & 2 & 99.92 & 100.00 \\
\hline 228 & 2053 & 2045 & 8 & 11 & 19 & 99.61 & 99.46 \\
\hline 230 & 2256 & 2256 & 0 & 0 & 0 & 100.00 & 100.00 \\
\hline 231 & 1571 & 1571 & 0 & 0 & 0 & 100.00 & 100.00 \\
\hline 232 & 1780 & 1780 & 0 & 6 & 6 & 100.00 & 99.66 \\
\hline 233 & 3079 & 3072 & 7 & 0 & 7 & 99.77 & 100.00 \\
\hline 234 & 2753 & 2752 & 1 & 0 & 1 & 99.96 & 100.00 \\
\hline Total & 109494 & 109294 & 200 & 200 & 400 & 99.82 & 99.82 \\
\hline
\end{tabular}


TABLE 2: QRS complex detection performance compared to several algorithms (based on [15, Table II]).

\begin{tabular}{|c|c|c|c|c|c|c|c|}
\hline QRS detector & ANN & $\mathrm{TP}$ & FN & FP & Error $[\%]$ & Se $[\%]$ & $+\mathrm{P}[\%]$ \\
\hline Li et al. [12] & 104182 & 104070 & 112 & 65 & 0.17 & 99.89 & 99.94 \\
\hline Saxena et al. [14] & 103763 & 103664 & 99 & 102 & 0.19 & 99.9 & 99.9 \\
\hline Bahoura et al. [16] & 109809 & 109635 & 184 & 135 & 0.29 & 99.83 & 99.88 \\
\hline Martínez et al. [15] & 109428 & 109208 & 220 & 153 & 0.34 & 99.80 & 99.86 \\
\hline This work & 109494 & 109294 & 200 & 200 & 0.37 & 99.82 & 99.82 \\
\hline OSEA [4] & $91284^{*}$ & 91105 & 179 & 180 & 0.39 & 99.80 & 99.80 \\
\hline Lee et al. [11] & $109486^{*}$ & $109151^{*}$ & 335 & 137 & 0.43 & 99.69 & 99.88 \\
\hline Hamilton and Tompkins [6] & 109267 & 108927 & 340 & 248 & 0.54 & 99.69 & 99.77 \\
\hline Zong et al. [7] & NA & NA & NA & NA & 0.58 & 99.65 & 99.77 \\
\hline Pan and Tompkins [2] & 109809 & 109532 & 277 & 507 & 0.71 & 99.75 & 99.54 \\
\hline Afonso et al. [21] & 90909 & 90535 & 374 & 406 & 0.86 & 99.59 & 99.56 \\
\hline Poli et al. [22] & 109963 & 109522 & 441 & 545 & 0.90 & 99.60 & 99.50 \\
\hline Kunzmann et al. [10] & 91283 & NA & NA & NA & 1.41 & 98.86 & 99.73 \\
\hline Aristotle SW [23] & 109428 & 107567 & 1861 & 94 & 1.79 & 98.30 & 99.91 \\
\hline
\end{tabular}

* The numbers are recalculated.

performance obtained utilizing data blocks of 700 samples, time delay of 20 milliseconds and eight data points for area calculation. Because of initial data resampling to $250 \mathrm{sam}$ ples per second, the obtained QRS complex locations were recalculated to match the 360 samples per second data rate of the original data and the attribute file. Then the performance was tested applying a 40 milliseconds delay to the MIT-BIH Arrhythmia Database. The first channel of all 48 two-channel records throughout their entire length was used for testing the performance of the algorithm.

The columns in Table 1 represent the record number (REC), the number of heartbeats in the record (AHBs), the number of correctly (true positive) (TP) detected heartbeats, the number of missed (not detected) (FN) heartbeats, the number of false positive (FP) detections, the sum of falsely detected and missed heartbeats $(\mathrm{FN}+\mathrm{FP})$, the sensitivity (Se $[\%])$, and the positive predictivity (+P [\%]), respectively.

The results in Table 1 were obtained by the algorithm modified for nonrepeating data reception through a serial link. The area calculation was performed on the data stream and then partitioned to data blocks. In order to enable block processing through the complete record, 1000 replicates of the last data sample were added to the end of all records. The algorithm was based on assumption that every block starts at the last detected QRS complex, therefore data processing starts 200 milliseconds after the block starts. This is also the case for the first data block where the first 200 milliseconds of data are not scanned for peaks, which leads to FN detections at the start of some records $(207,208,210,214,220$, and 233).

The algorithm performs well and stable on all tested records (Table 1); however, performance on two records is far worse than on other records. The record 108 is problematic for many detection algorithms because of the first-degree AV block and high and sharp P waves. The combination of these two properties allows the $\mathrm{P}$ waves to qualify as the QRS complexes and produce FP detections in several places in the record. Detection performance on the record 203 mainly suffers from nondetected (FN) beats, mostly premature ventricular contractions (PVCs). The nondetected PVCs have low amplitude and occur between two normal high-amplitude beats. In such conditions, these beats either do not exceed any of the detection threshold levels or exceed the lower detection threshold level, but there is no search-back.

To gain the sense of the algorithm performance on the MIT-BIH Arrhythmia Database, a comparison of detection results to a set of other well-known published algorithms is reported in Table 2. The columns in Table 2 represent the QRS detector (QRS detector), the number of tested (annotated) (ANN) heartbeats, the number of correctly (true positive) (TP) detected heartbeats, the number of missed (not detected) (FN) heartbeats, the number of false positive (FP) detections, percentage of falsely detected and missed heartbeats among all tested heartbeats (Error \%), the sensitivity $($ Se $[\%])$, and the positive predictivity $(+\mathrm{P}[\%])$, respectively.

With Se of $99.82 \%$ and $+\mathrm{P}$ of $99.82 \%$ the algorithm performs satisfactory when compared to other algorithms, and outperforms the founding algorithm [11]. When the two algorithms are compared, it is interesting to see how the detection performance is influenced by different filtering and detection threshold level calculation. While our algorithm exhibits worse detection performance on, for example, records 104 and 108, it performs much better on, for example, record 222. We are confident that further algorithm improvements would lead to even better QRS complex detection results. An improvement in detection threshold level calculation could prevent sudden baseline shifts or some QRS complexes from raising the detection threshold level to a point where no QRS is detected in a subsequent block, which in the record 203 happens at 108.4 seconds. Furthermore, incorporation of a positive-negative wave pair detection mechanism similar to other high-performance detection algorithms would exclude sudden baseline shifts from the QRS candidate list. 
TABle 3: Performance of the algorithm on 43 records of the LTST DB.

\begin{tabular}{|c|c|c|c|c|c|c|c|c|}
\hline REC & AHB & THB & $\mathrm{TP}$ & $\mathrm{FN}$ & $\mathrm{FP}$ & $\mathrm{FN}+\mathrm{FP}$ & Se [\%] & $+\mathrm{P}[\%]$ \\
\hline s20011 & 100053 & 99674 & 99670 & 4 & 0 & 4 & 100.00 & 100.00 \\
\hline s20021 & 88963 & 88598 & 88597 & 1 & 5 & 6 & 100.00 & 99.99 \\
\hline s20031 & 109501 & 109109 & 109095 & 14 & 25 & 39 & 99.99 & 99.98 \\
\hline s20041 & 109304 & 108993 & 108977 & 16 & 0 & 16 & 99.99 & 100.00 \\
\hline s20061 & 117925 & 117454 & 117451 & 3 & 1 & 4 & 100.00 & 100.00 \\
\hline s20081 & 112979 & 112553 & 112553 & 0 & 1 & 1 & 100.00 & 100.00 \\
\hline s20101 & 78017 & 77649 & 77649 & 0 & 21 & 21 & 100.00 & 99.97 \\
\hline s20121 & 85526 & 85220 & 85210 & 10 & 6 & 16 & 99.99 & 99.99 \\
\hline s20141 & 116674 & 116206 & 116206 & 0 & 0 & 0 & 100.00 & 100.00 \\
\hline s20161 & 83698 & 83355 & 83337 & 18 & 226 & 244 & 99.98 & 99.73 \\
\hline s20181 & 106978 & 106555 & 106498 & 57 & 150 & 207 & 99.95 & 99.86 \\
\hline s20201 & 91477 & 91058 & 91057 & 1 & 1 & 2 & 100.00 & 100.00 \\
\hline s20221 & 119182 & 118715 & 118714 & 1 & 0 & 1 & 100.00 & 100.00 \\
\hline s20241 & 92439 & 91997 & 91997 & 0 & 0 & 0 & 100.00 & 100.00 \\
\hline s20261 & 102311 & 101955 & 101953 & 2 & 0 & 2 & 100.00 & 100.00 \\
\hline s20281 & 73076 & 72808 & 72807 & 1 & 1 & 2 & 100.00 & 100.00 \\
\hline s20301 & 106779 & 106379 & 106378 & 1 & 3 & 4 & 100.00 & 100.00 \\
\hline s20321 & 91929 & 91586 & 91542 & 44 & 18 & 62 & 99.95 & 99.98 \\
\hline s20341 & 100255 & 99816 & 99814 & 2 & 7 & 9 & 100.00 & 99.99 \\
\hline s20361 & 105688 & 105380 & 105377 & 3 & 37 & 40 & 100.00 & 99.96 \\
\hline s20381 & 102972 & 102884 & 102883 & 1 & 10 & 11 & 100.00 & 99.99 \\
\hline s20401 & 77333 & 77048 & 77046 & 2 & 30 & 32 & 100.00 & 99.96 \\
\hline s20421 & 92966 & 92582 & 92578 & 4 & 2 & 6 & 100.00 & 100.00 \\
\hline s20441 & 93127 & 92737 & 92735 & 2 & 54 & 56 & 100.00 & 99.94 \\
\hline s20461 & 98872 & 98489 & 98487 & 2 & 0 & 2 & 100.00 & 100.00 \\
\hline s20481 & 91455 & 91117 & 91027 & 90 & 288 & 378 & 99.90 & 99.68 \\
\hline s20501 & 142725 & 142207 & 142175 & 32 & 26 & 58 & 99.98 & 99.98 \\
\hline s20521 & 75336 & 74957 & 74918 & 39 & 723 & 762 & 99.95 & 99.04 \\
\hline s20541 & 115151 & 114375 & 114354 & 21 & 84 & 105 & 99.98 & 99.93 \\
\hline s20561 & 100816 & 100391 & 100370 & 21 & 21 & 42 & 99.98 & 99.98 \\
\hline s20581 & 84935 & 84601 & 84596 & 5 & 5 & 10 & 99.99 & 99.99 \\
\hline s20601 & 116943 & 116488 & 116365 & 123 & 149 & 272 & 99.89 & 99.87 \\
\hline s20621 & 112369 & 111933 & 111829 & 104 & 2320 & 2424 & 99.91 & 97.97 \\
\hline s20641 & 84854 & 84426 & 84423 & 3 & 44 & 47 & 100.00 & 99.95 \\
\hline s30661 & 144447 & 143932 & 143925 & 7 & 26 & 33 & 100.00 & 99.98 \\
\hline s30681 & 126651 & 126101 & 126097 & 4 & 145 & 149 & 100.00 & 99.89 \\
\hline s30701 & 107078 & 106719 & 106715 & 4 & 4 & 8 & 100.00 & 100.00 \\
\hline s30721 & 106636 & 106234 & 105818 & 416 & 7212 & 7628 & 99.61 & 93.62 \\
\hline s30741 & 123461 & 123064 & 122984 & 80 & 2 & 82 & 99.93 & 100.00 \\
\hline s30742 & 113767 & 113317 & 113311 & 6 & 0 & 6 & 99.99 & 100.00 \\
\hline s30761 & 117044 & 116677 & 115883 & 794 & 313 & 1107 & 99.32 & 99.73 \\
\hline s30781 & 110087 & 109635 & 109231 & 404 & 5359 & 5763 & 99.63 & 95.32 \\
\hline s30801 & 94373 & 94055 & 84133 & 9922 & 10559 & 20481 & 89.45 & 88.85 \\
\hline Total & 4426152 & 4409029 & 4396765 & 12264 & 27878 & 40142 & 99.72 & 99.37 \\
\hline
\end{tabular}

We then additionally tested the developed algorithm on the 43 publicly accessible records [25] of the LTST DB. The complete database contains 86 two- and three-channel 24hour annotated ambulatory records, where some records present great difficulties for accurate QRS complex detection. The LTST DB contains records contaminated with lots of noises, arrhythmias, and in addition to this, also severe transient ischemic changes. The algorithm was tested on the first channel of each of the 43 records with the first five minutes of each record excluded from performance analysis. In this way, the algorithm performance was tested on 4409029 out of 4426152 beats and the results for QRS 
detection Se and $+\mathrm{P}$ were $99.72 \%$ and $99.37 \%$, respectively, as shown in Table 3. The performance of the OSEA algorithm on the same data set is $99.79 \%$ for Se and $99.37 \%$ for $+\mathrm{P}$. The columns in Table 3 represent the record number (REC), the number of all heartbeats in the record (AHBs), the number of tested heartbeats (THBs), the number of correctly (true positive) (TP) detected heartbeats, the number of missed (not detected) (FN) heartbeats, the number of false positive detections (FP), the sum of falsely detected and missed heartbeats $(\mathrm{FN}+\mathrm{FP})$, the sensitivity $($ Se $[\%])$, and the positive predictivity $(+\mathrm{P}[\%])$, respectively.

We tried to compare our work with other works in this field, but there is lack of papers that describe detection results of modified QRS complex detection algorithms and their HW implementations. Therefore, our work can only be paralleled to $[26,27]$. Two HW implementations of a QRS complex detector are described in [26]. One implementation is a modification of the well-known Hamilton-Tompkins [6] detector and the other is a single-scale $\left(W_{2}{ }^{4}\right)$ WT-based QRS complex detector. Only modest performance results were given and only descriptive comparisons of the results were made to [13]. As both $[13,26]$ were tested on the AHA database [28], we could not compare the performance of those two algorithms with the performance of our algorithm. When the size and speed of the design are compared to the design in [26], large differences can be seen. The QRS complex detection algorithm in [26] uses less than $1 \%$ of the Xilinx XC2V3000-6 [24] device resources and can operate at $34 \mathrm{MHz}$. Our QRS complex detection algorithm together with all communication logic uses approximately $17 \%$ of the XC2V3000 device resources, but the estimated operating speed of the device is $82 \mathrm{MHz}$. In [27], a lifting WTbased FPGA implementation of a QRS complex detection algorithm is presented, but no accurate performance results are supplied. While these two works present pure HW solutions to QRS complex detection (except coefficient prelearning in [27]), the HW/SW solution in [29] uses the FPGA as a coprocessor engine for cross-correlation coefficient calculation.

An important feature of the HW implementation of the algorithm is data processing speed. To obtain the most comparable results of both HW and SW implementations, a comparison of block processing speed in the HW and SW implementations was made. The time needed to find all peaks in one block and determine QRS complex locations was measured. In average, approximately $132 \mu$ s were required by the HW implementation to perform these operations, while for the SW implementation in average it took approximately $120 \mu$ s to perform the same operations. It needs to be singled out that the operating frequency of the HW implementation was only $5 \mathrm{MHz}$, while the SW implementation ran on an AMD Athlon 2500+ processor with the operating frequency of $1.8 \mathrm{GHz}$. Taking into consideration that the HW implementation can operate at $80 \mathrm{MHz}$, we can conclude that the HW implementation can process data up to 14 times faster than SW implementation. This means that in the same time the HW device can process up to 14 times more data than a PC.

\section{CONCLUSION}

An ECG beat detection algorithm based on delay-coordinate mapping was presented in this paper. Heartbeat detection was based on the size of the area created in a 2D geometrical plane by the ECG signal and its time delayed copy. The algorithm processes blocks of data and is applicable in systems where true real-time beat detection is not needed, but the data can rather be grouped into blocks and then processed. Outperforming the founding algorithm, the QRS complex detection performance of the proposed algorithm is comparable to other detection algorithms. With Se of $99.82 \%$ and $+\mathrm{P}$ of $99.82 \%$ on the MIT-BIH Arrhythmia Database the algorithm is superior to a large number of well-known algorithms. The performance was additionally tested on the LTST DB, where Se of $99.72 \%$ and $+\mathrm{P}$ of $99.37 \%$ were achieved. The algorithm was implemented in both SW and HW, where with all communication logic and without any code optimization it fits into the Xilinx XC3S400 device. Besides good detection results, the HW implementation of the algorithm excels itself with high data processing speed and the possibility of multiplying data processing cores in a single FPGA device. Being 14 times faster than the SW solution, the HW solution represents a valuable contribution to ECG signal processing, either as an easily upgradeable stand-alone beat detector or as a very powerful coprocessing engine.

\section{REFERENCES}

[1] B.-U. Köhler, C. Hennig, and R. Orglmeister, "The principles of software QRS detection," IEEE Engineering in Medicine and Biology Magazine, vol. 21, no. 1, pp. 42-57, 2002.

[2] J. Pan and W. J. Tompkins, "A real-time QRS detection algorithm," IEEE Transactions on Biomedical Engineering, vol. 32, no. 3, pp. 230-236, 1985.

[3] N. V. Thakor, J. G. Webster, and W. J. Tompkins, "Estimation of QRS complex power spectra for design of a QRS filter," IEEE Transactions on Biomedical Engineering, vol. 31, no. 11, pp. 702-706, 1984.

[4] P. S. Hamilton, Open Source ECG Analysis Software Documentation, E. P. Limited, Somerville, Mass, USA, 2002.

[5] R. G. Mark, P. S. Schluter, G. B. Moody, P. Devlin, and D. Chernoff, "An annotated ECG database for evaluating arrhythmia detectors," in Proceedings of the 4th Annual Conference of IEEE Engineering in Medicine and Biology Society on Frontiers of Engineering in Health Care, pp. 205-210, IEEE Computer Society Press, Philadelphia, Pa, USA, 1982.

[6] P. S. Hamilton and W. J. Tompkins, "Quantitative investigation of QRS detection rules using the MIT/BIH arrhythmia database," IEEE Transactions on Biomedical Engineering, vol. 33, no. 12, pp. 1157-1165, 1986.

[7] W. Zong, G. B. Moody, and D. Jiang, "A robust open-source algorithm to detect onset and duration of QRS complexes," in Computers in Cardiology, vol. 30, pp. 737-740, Thessaloniki, Chalkidiki, Greece, September 2003.

[8] I. I. Christov and T. V. Stoyanov, "Steep slope method for real time QRS detection," Electrotechnics \& Electronics E+E, vol. 12, pp. 13-17, 2002.

[9] I. I. Christov, "Real time electrocardiogram QRS detection using combined adaptive threshold," Biomedical Engineering Online, vol. 3, no. 1, p. 28, 2004. 
[10] U. Kunzmann, G. von Wagner, J. Schöchlin, and A. Bolz, "Parameter extraction of ECG signals in real-time," Biomedizinische Technik, vol. 47, supplement 1 part 2, pp. 875-878, 2002.

[11] J.-W. Lee, K.-S. Kim, B. Lee, B. Lee, and M.-H. Lee, "A real time QRS detection using delay-coordinate mapping for the microcontroller implementation," Annals of Biomedical Engineering, vol. 30, no. 9, pp. 1140-1151, 2002.

[12] C. Li, C. Zheng, and C. Tai, "Detection of ECG characteristic points using wavelet transforms," IEEE Transactions on Biomedical Engineering, vol. 42, no. 1, pp. 21-28, 1995.

[13] S. Kadambe, R. Murray, and G. F. Boudreaux-Bartels, "Wavelet transform-based QRS complex detector," IEEE Transactions on Biomedical Engineering, vol. 46, no. 7, pp. 838-848, 1999.

[14] S. C. Saxena, V. Kumar, and S. T. Hamde, "Feature extraction from ECG signals using wavelet transforms for disease diagnostics," International Journal of Systems Science, vol. 33, no. 13, pp. 1073-1085, 2002.

[15] J. P. Martínez, R. Almeida, S. Olmos, A. P. Rocha, and P. Laguna, "A wavelet-based ECG delineator: evaluation on standard databases," IEEE Transactions on Biomedical Engineering, vol. 51, no. 4, pp. 570-581, 2004.

[16] M. Bahoura, M. Hassani, and M. Hubin, "DSP implementation of wavelet transform for real time ECG wave forms detection and heart rate analysis," Computer Methods and Programs in Biomedicine, vol. 52, no. 1, pp. 35-44, 1997.

[17] F. Jager, A. Taddei, G. B. Moody, et al., "Long-term ST database: a reference for the development and evaluation of automated ischaemia detectors and for the study of the dynamics of myocardial ischaemia," Medical and Biological Engineering and Computing, vol. 41, no. 2, pp. 172-182, 2003.

[18] D. L. Perry, VHDL: Programming by Example, McGraw-Hill, New York, NY, USA, 2002.

[19] P. A. Lynn, "Online digital filters for biological signals: some fast designs for a small computer," Medical and Biological Engineering and Computing, vol. 15, no. 5, pp. 534-540, 1977.

[20] F. Jager, "QRS complex detection in electrocardiogram," M.S. thesis, University of Ljubljana, Ljubljana, Slovenia, 1984.

[21] V. X. Afonso, W. J. Tompkins, T. Q. Nguyen, and S. Luo, "ECG beat detection using filter banks," IEEE Transactions on Biomedical Engineering, vol. 46, no. 2, pp. 192-202, 1999.

[22] R. Poli, S. Cagnoni, and G. Valli, "Genetic design of optimum linear and nonlinear QRS detectors," IEEE Transactions on Biomedical Engineering, vol. 42, no. 11, pp. 1137-1141, 1995.

[23] G. B. Moody and R. G. Mark, "Development and evaluation of a 2-lead ECG analysis program," in Computers in Cardiology, pp. 39-44, Seattle, Wash, USA, 1983.

[24] Xilinx, http://www.xilinx.com/.

[25] "Long-Term ST Database at Physionet," http://physionet.org/ physiobank/database/ltstdb/.

[26] M. M. Peiró, F. Ballester, G. Paya, J. Belenguer, R. Colom, and R. Gadea, "FPGA custom DSP for ECG signal analysis and compression," in Proceedings of the 14th International Conference on Field Programmable Logic and Application (FPL '04), vol. 3203 of Lecture Notes in Computer Science, pp. 954-958, Leuven, Belgium, August-September 2004.

[27] K. Kuzume, K. Niijima, and S. Takano, "FPGA-based lifting wavelet processor for real-time signal detection," Signal Processing, vol. 84, no. 10, pp. 1931-1940, 2004.

[28] R. E. Hermes, D. B. Geselowitz, and G. C. Oliver, "Development, distribution, and use of the American heart association database for ventricular arrhythmia detector evaluation," in Computers in Cardiology, pp. 263-266, Williamsburg, Va, USA, October 1980
[29] A. Rosado, M. Bataller, J. F. Guerrero, J. Calpe, J. V. Francés, and J. R. Magdalena, "High performance hardware correlation coefficient assessment using programmable logic for ECG signals," Microprocessors and Microsystems, vol. 27, no. 1, pp. 3339, 2003.

Matej Cvikl received his B.S. degree in electrical engineering from the Faculty of Electrical Engineering, University of Ljubljana, in 2002. Since then, he has been working as HW and System Design Engineer. In October 2004, he started working towards the Ph.D. degree at the Faculty of Electrical Engineering, supported by Ministry of Higher Education, Science and Technology. His current research includes HW/SW codesign in the area of ECG signal processing on FPGAs.

Franc Jager received a Ph.D. degree in computer and information science from the University of Ljubljana, in 1994. Currently, he is a Full Professor in the Faculty of Computer and Information Science at the University of Ljubljana, and a Research Affiliate at the Massachusetts Institute of Technology, Cambridge. His research interests include biomedical signal processing and medical imaging, and biomedical computer systems.

Andrej Zemva received his B.S., M.S., and Ph.D. degrees in electrical engineering from the University of Ljubljana, in 1989, 1993, and 1996, respectively. He is Associate Professor at the Faculty of Electrical Engineering. His current research interests include HW/SW codesign, logic synthesis and optimization, test pattern generation, and fault modeling.
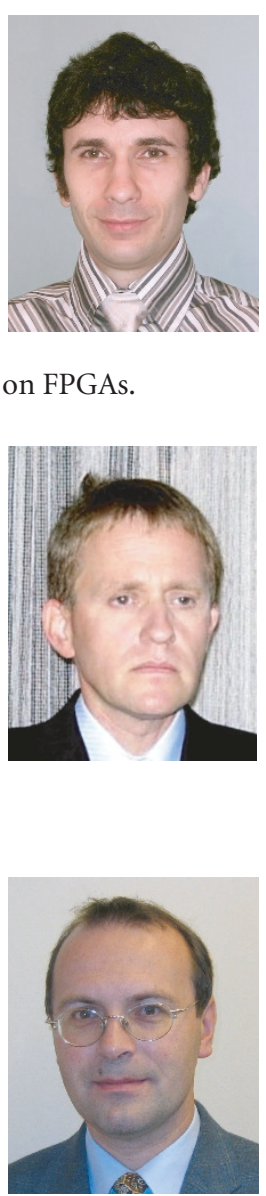